\title{
A study of the nonlinearity of a building thermal behavior based on metamodeling
}

\author{
Issa Jaffal $^{1}$, and Christian Inard ${ }^{2, *}$ \\ ${ }^{1}$ Laboratoire du Froid, des Systèmes Energétiques et Thermiques (LaFSET), Cnam, HESAM Université, 75003 Paris, France \\ ${ }^{2}$ LaSIE, University of La Rochelle, 17000 La Rochelle, France
}

\begin{abstract}
In this work, we present a study of the nonlinearity of building thermal behavior based on a metamodel for cooling energy needs. We studied the nonlinearity of the thermal behavior of an office. The building quadratic behavior and interactions between its components were analyzed based on the metamodel coefficients. The metamodel was fitted with a reduced number of dynamic simulations. The nonlinearity was first assessed as function of the mean outdoor air temperature in fifteen typical European climates and then as function of the internal heat gains for the coldest and hottest climates. The metamodel provided highly accurate results with fast calculation time. However, a higher accuracy was generally obtained for hot climates, high internal heat gains and lightweight thermal mass. Conversely, the nonlinearity of thermal behavior was accentuated in cold climates and with low internal heat gains. Moreover, the interactions between the building components were found to be more influential on cooling energy needs than quadratic behavior. We propose a classification of thermal behavior into three regimes: Highly nonlinear when the energy needs are close to zero; intermediate with decreasing nonlinearities that can be expressed by power functions; and finally, a quasi-linear regime with almost-steady nonlinearities.
\end{abstract}

\section{Introduction}

Dynamic models describe the thermal behavior of a building with relatively high fidelity and can be used to explore design alternatives. However, despite their great potential, extensive studies such as those for building optimization, may require excessive computation times. In addition, their capacity to provide insight into thermal behavior is limited by the implicit nature of the heat transfer equations. Furthermore, their use during early design stages is constrained by the large amount of data required.

Metamodels have been developed to approximate simulation models [1], with a resulting improvement in computation efficiency and a better understanding of the original model. The simulations are simplified by the low computational expense of the metamodels. In addition, metamodels generally have explicit forms which provide insight into the nature of the simulation response as a function of the influential parameters. Moreover, they only require small amounts of data, which makes them suitable for early design stages

The most common metamodeling strategy is to construct polynomial approximations $[2,3]$. They are the simplest, require the lowest computational effort and give useful insight into the behavior of the model. Their coefficients are easy to interpret, highlighting the effects of the input parameters. The number of runs needed to fit them can be drastically reduced with the use of the Design of Experiments method [4]. The choice of an experimental design determines the number of runs and the value of the design parameters in each run.

Several alternative metamodeling techniques can be used to approximate a model, notably artificial neural networks, radial basis functions, kriging, Multivariate Adaptive Regression Splines (MARS), support vector machines and Gaussian processes [2, 3].

Generally, these techniques provide better fits than polynomials. However, they can be computationally intensive and provide less insight.

Hence, metamodels can improve the computation efficiency of building energy performance. They can also provide an interpretation of the dynamic model, especially when using polynomials. This allows a better understanding of the relationship between building design, environmental parameters and energy performance indicators.

Consequently, the development of metamodels to investigate building energy performance has become an active area of research. Polynomial regressions are the most widely used metamodels. They have been used to study the energy needs for cooling and heating [5-7], energy consumption [8-10] and $\mathrm{CO}_{2}$ emissions [7].

Moreover, polynomial regressions have been developed to assess the impact of building energy consumption with climate change [11] and to estimate the energy consumption of a building stock [12].

Artificial neural networks have been also widely used for building energy performance metamodeling [13-15]. Furthermore, various metamodeling techniques have

\footnotetext{
* Corresponding author: christian.inard@univ-lr.fr
} 
been investigated to study building energy performance including support vector machines [16, 17], Gaussian process [18] and MARS [19, 20].

Finally, metamodels have recently been introduced in Brazilian energy regulations to assess the energy performance of air-conditioned and naturally ventilated buildings [10], and they have been recommended for future Chilean energy standards [7].

The thermal behavior of a building is affected by nonlinearities. Heat transfer through walls is nonlinear in a transient regime. Heat transfer by convection and radiation is inherently nonlinear, although it is often linearized. The thermal behavior of the energy systems of a building is generally nonlinear.

There are also interactions between heat transfers because the effect of one heat transfer on energy needs can depend on the level of another. For instance, the effect of a ventilation heat transfer in reducing the energy needs for cooling is greater when the solar and internal gains are high.

Consequently, understanding the nonlinearities in the thermal behavior of a building and assessing the relevance of linear calculation methods are interesting subjects for research. Nonlinearity has been investigated by simply changing one variable at time, such as in studying the impact of passive design measures on the building energy consumption for heating and cooling [21], the impact of uncertainties in building parameters on energy and economic performance [22] and energy consumption as a function of the U-values of [23].

Nonlinearity has also been highlighted in sensitivity analysis studies using the Morris method, for instance in the sensitivity analysis of the heating energy needs with respect to building parameters [24] and of the heating energy demand and overheating hours with respect to weather variables [25].

Comparative studies have been conducted to confront linear and nonlinear regression metamodels, highlighting the higher accuracy of the latter. For instance, linear and quadratic regression metamodels have been developed to predict energy consumption as a function of the building and HVAC system parameters [26]. However, the literature shows that there is a need for a general and simple method to assess the nonlinearities of thermal behavior.

Thus, extensive work has been carried out on metamodels for the study of the energy performance of a building and its energy systems. These studies emphasized the accuracy and computational efficiency of metamodels, but their capability to provide insight into thermal behavior has not been sufficiently investigated. In particular, there is a lack of methods that use metamodels to study nonlinearities in thermal behavior.

Recently, we presented a general metamodel that can be used as a common framework for metamodeling building energy performance [27]. Here we present a method for the study of nonlinearities in the thermal behavior of a building based on a metamodel for the cooling energy needs derived from the general one.

In order to assess these nonlinearities, we introduced two measures based on the metamodel coefficients highlighting the importance of the quadratic and interaction effects.

The method was applied to analyze the nonlinearity of the thermal behavior of an office for fifteen typical European climates. Moreover, the method was applied to the cold climate of Helsinki and the hot climate of Athens with various levels of internal heat gains.

\section{Methods}

\subsection{Metamodeling}

The metamodel was derived from the general metamodel for building energy performance that we presented previously [27]. The derivation was achieved by considering the cooling energy needs $Q_{c}$ as a performance indicator. Hence, the energy needs are a second-order polynomial of the individual energy needs for cooling $\mathbf{Q}=\left(Q_{1}, Q_{2}, \ldots, Q_{n}\right)$ of the building components, which is expressed as follows:

$$
\begin{aligned}
& Q_{c}=a_{0}+\sum_{i=1}^{n} a_{i} Q_{i}+ \\
& \sum_{i=1}^{n} a_{i i} Q_{i}^{2}+\sum_{i=1}^{n-1} \sum_{j=i+1}^{n} a_{i j} Q_{i} Q_{j}+\varepsilon
\end{aligned}
$$

where $Q_{i}$ and $Q_{j}$ are two individual energy needs equal to two heat transfers ( $\mathrm{kWh}$ year $\left.^{-1}\right), a_{0}, a_{i}, a_{i i}$, and $a_{i j}$ are the linear, quadratic and interaction effects, respectively and $\varepsilon$ is the residual.

The coefficients $a_{0}, a_{i}, a_{i i}$, and $a_{i j}$ are assumed to depend on climate, the thermal mass of the building, its use, the type of energy system and possibly the individual energy needs that are assumed to be constant. When all the individual energy needs of all the building components vary, $a_{0}$ is equal to zero.

A transmission energy need for a wall is calculated, in a quasi-steady-state assumption as

$$
Q_{t r}=\sum U A\left(\theta_{i s}-\theta_{o e}\right) \Delta t
$$

where $U$ and $A$ are the $\mathrm{U}$-value $\left(\mathrm{W} \mathrm{m}^{-2} \mathrm{k}^{-1}\right)$ and the area of the wall $\left(\mathrm{m}^{2}\right)$, respectively, $\theta_{i s}$ is the indoor setpoint temperature $\left({ }^{\circ} \mathrm{C}\right), \theta_{o e}$ is the equivalent outdoor temperature to which the wall is exposed $\left({ }^{\circ} \mathrm{C}\right)$ and $\Delta t$ is the time step (h).

The energy need of an air change is given by

$$
Q_{a c}=\sum \rho_{a} c_{p a} q_{v, a c}\left(\theta_{i s}-\theta_{o a}\right) \Delta t
$$

where $q_{v, a c}$ is the airflow rate $\left(\mathrm{m}^{3} \mathrm{~s}^{-1}\right), \rho_{a}$ and $c_{p a}$ are the air density $\left(\mathrm{kg} \mathrm{m}^{-3}\right)$ and specific heat capacity $\left(\mathrm{J} \mathrm{kg}^{-1}\right.$ $\left.\mathrm{k}^{-1}\right)$, respectively, $\theta_{o a}$ is the outdoor air temperature $\left({ }^{\circ} \mathrm{C}\right)$.

The individual energy need of a solar heat gain through a window can be expressed as

$$
Q_{s o}=\sum F_{i s h} F_{e s h} S H G C_{w} I_{s w} A_{w} \Delta t
$$

where $S H G C_{w}$ and $A_{w}$ are the solar heat gain coefficient and the area of the window $\left(\mathrm{m}^{2}\right)$, respectively, $I_{s w}$ is the solar irradiance in the direction of the window $\left(\mathrm{W} \mathrm{m}^{-2}\right)$ and $F_{\text {ish }}$ and $F_{\text {esh }}$ are the shading reduction factors of the internal and external shading devices, respectively. 


\subsection{Nonlinearity measures}

The nonlinearities in thermal behavior were analyzed based on the interpretation of the metamodel coefficients. To this end, we introduce the following measures, which quantify the importance of the quadratic and the interaction effects.

The ratio of the quadratic to the linear effects is given by

$$
Q L=\frac{\sum_{i=1}^{n} \mid a_{i i}}{\sum_{i=1}^{n}\left|a_{i}\right|}
$$

where $a_{i}$ is the effect of a linear term, $a_{i j}$ is the effect of a quadratic term and $n$ is the number of linear terms equal to the number of the quadratic terms.

The ratio of the interaction to the linear effects is calculated from

$$
I L=\frac{n_{i} \sum_{i=1}^{n-1} \sum_{j=i+1}^{n}\left|a_{i j}\right|}{n_{i j} \sum_{i=1}^{n}\left|a_{i}\right|}
$$

where $a_{i j}$ is the effect of an interaction term and $n_{i j}=n_{i}\left(n_{i}-1\right) / 2$ is the number of interaction terms.

\subsection{Case study}

The metamodel for cooling energy needs was used to study the nonlinearity of the thermal behavior of the office shown in Fig. 1. The energy needs were assessed for the period from June to September. The office has a concrete structure.

Two types of thermal mass were considered: a lightweight thermal mass with insulation from the inside, and a heavy thermal mass with insulation from the outside.

The office is occupied from Monday to Friday from $8 \mathrm{~h}$ to $18 \mathrm{~h}$. The ventilation air flow is equal to $50 \mathrm{~m}^{3} \mathrm{~h}^{-1}$ when the office is occupied. The cooling set-point temperatures is $26{ }^{\circ} \mathrm{C}$ when the office is occupied and $30^{\circ} \mathrm{C}$ when it is unoccupied.

Moreover, the basic value of the internal heat gains is $20 \mathrm{~W} \mathrm{~m}^{-2}$ when the office is occupied and $2 \mathrm{~W} \mathrm{~m}^{-2}$ when it is unoccupied.

We studied the impact of the facade components on cooling energy needs. Consequently, we analyzed individual energy needs corresponding to four heat transfers. These are shown in Table 1, with the parameters in Eqs. (2)-(4) varied to fit the metamodel.

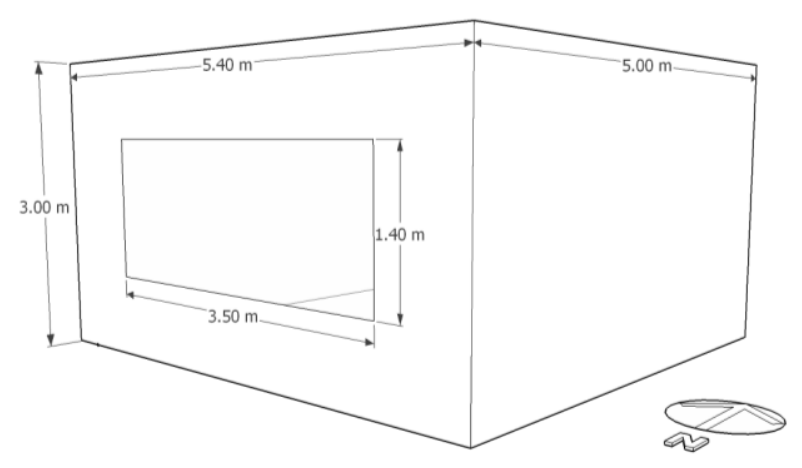

Fig. 1. The studied office.

Moreover, an external shading device was included. It provides a shading factor $F_{o m}=0.2$ when the solar irradiance $I_{s w}$ is higher than $300 \mathrm{~W} \mathrm{~m}^{-2}$.

The metamodel was fitted from dynamic simulations performed with TRNSYS software. To this end, the individual energy needs were varied using upper and lower levels of the physical parameters, as shown in Table 2.

The Box-Behnken experimental design was used to plan the simulations [28]. Hence, 25 dynamic simulations were needed to fit the metamodel compared with 81 when using a full factorial design. In addition, the metamodel coefficients were obtained by multiple regression analysis.

Next, the metamodel fit was tested by comparing the results with those of the TRNSYS dynamic simulations. The comparison was performed for 100 additional dynamic simulations with a random combination of the physical parameters of Table 2 .

Once the metamodel had been fitted and validated, the nonlinearities in thermal behavior were studied using the measures of Eqs. (5)-(6). The values of these measures were calculated using coded variables of the individual energy needs ranging from -1 to +1 . The nonlinearities were hence analyzed using individual energy needs having the same variation range.

The metamodel was first applied to fifteen typical European climates, then for the cold climate of Helsinki and the hot climate of Athens.

In the second part, the cooling energy needs and the nonlinearities in thermal behavior were studied in relation to internal heat gains $p_{i g, o}$ of between 5 and 40 $\mathrm{W} \mathrm{m} \mathrm{m}^{-2}$ during the occupation, at increments of $5 \mathrm{~W} \mathrm{~m}^{-2}$. In addition, internal heat gains when unoccupied were $10 \%$ of $p_{\text {ig,o. }}$.

In each case, the mean value of cooling energy needs was considered to be equal to the mean of the 100 dynamic simulations used to test the metamodel fit.

Table 1. Heat transfers, individual energy needs and the parameters varied to fit the metamodel.

\begin{tabular}{|c|c|c|}
\hline Heat transfer & Individual energy need & Varied parameter \\
\hline $\begin{array}{c}\text { Transmission through the opaque wall } \\
\text { incorporating the effect of the thermal bridges }\end{array}$ & $Q_{t r, w o}\left(\mathrm{kWh} \mathrm{year-1}^{-1}\right)$ & $U_{o w}\left(\mathrm{~W} \mathrm{~m}^{-2} \mathrm{~K}^{-1}\right)$ \\
\hline Transmission through the window & $Q_{t r, w}\left(\mathrm{kWh} \mathrm{year}^{-1}\right)$ & $U_{w}\left(\mathrm{~W} \mathrm{~m}^{-2} \mathrm{~K}^{-1}\right)$ \\
\hline Solar heat gain through the window & $Q_{s o, w}\left(\mathrm{kWh} \mathrm{year}^{-1}\right)$ & $q_{v, \text { inf }}\left(\mathrm{m}^{3} \mathrm{~h}^{-1}\right)$ \\
\hline Heat transfer due to infiltration & $Q_{a c, \text { inf }}\left(\mathrm{kWh} \mathrm{year}^{-1}\right)$ & $S H G C_{w}$ \\
\hline
\end{tabular}




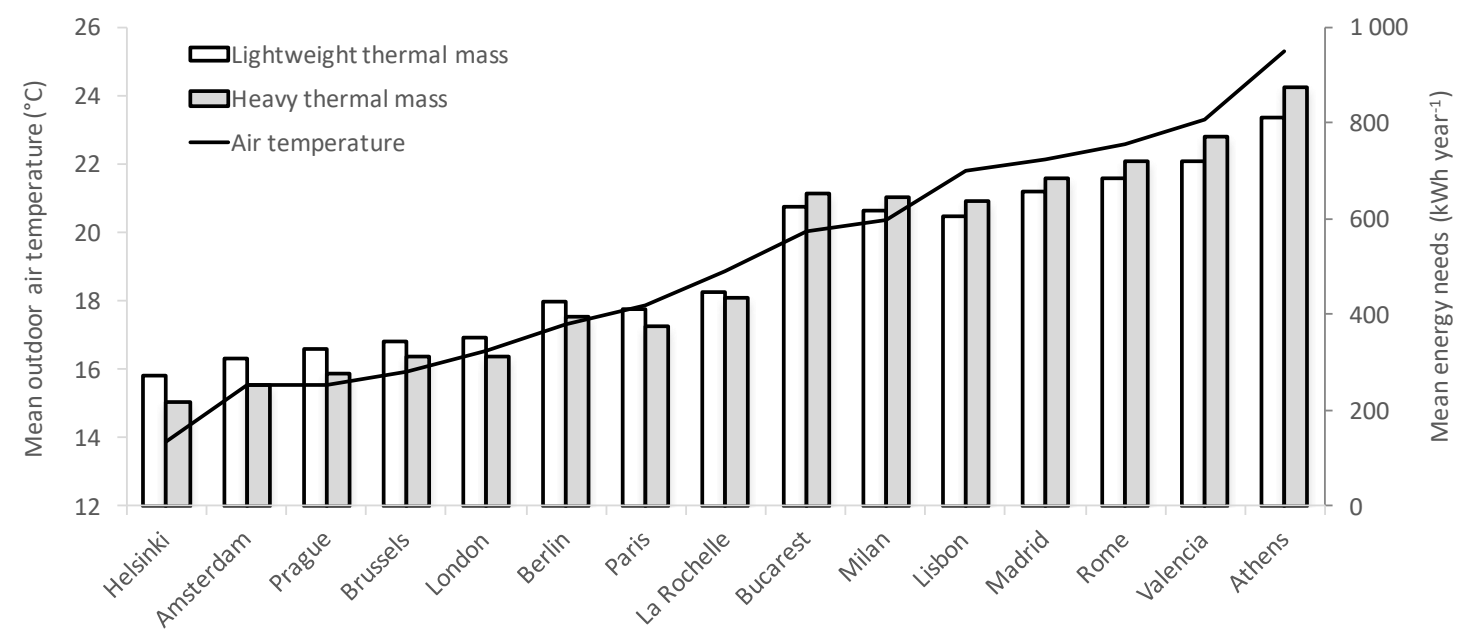

Fig. 2. Mean outdoor air temperature and mean cooling energy needs for fifteen typical European climates.

Table 2. Lower and upper levels of the physical parameters.

\begin{tabular}{|c|c|c|c|c|}
\hline Para & $\begin{array}{c}U_{o w} \\
\left(\mathbf{W} \mathbf{m}^{-2} K^{-1}\right)\end{array}$ & $\begin{array}{c}U_{w} \\
\left(\mathbf{W} \mathbf{m}^{-2} \mathbf{K}^{-1}\right)\end{array}$ & $\begin{array}{c}q_{v, i n f} \\
\left(\mathbf{m}^{3} \mathbf{h}^{-1}\right)\end{array}$ & $\begin{array}{c}S H G C_{w} \\
-\end{array}$ \\
\hline Lower & 0.1 & 0.7 & 8.1 & 0.3 \\
\hline Upper level & 0.5 & 2.7 & 32.4 & 0.7 \\
\hline
\end{tabular}

\section{Results and discussions}

\subsection{Application to typical European climates}

As specified previously, the metamodel was applied to fifteen typical European climates. The mean outdoor air temperature in the corresponding locations and the mean cooling energy needs are presented in Fig. 2. The cooling energy needs varied between 271.1 and 810.9 $\mathrm{kWh}$ year $^{-1}$ for the lightweight thermal mass and between 215.3 and $871.8 \mathrm{kWh}$ year $^{-1}$ for the heavy thermal mass. In addition, the results indicate that the thermal mass decreased the energy needs in cold climates and increased it in hot climates.

The variation of the root mean square error (RMSE) of the metamodel versus the mean outdoor air temperature in the different locations for the lightweight and heavy thermal masses is presented in Fig. 3. The $R M S E$ generally decreased with the temperature and thermal mass. Moreover, the RMSE varied between 0.4 and $1.4 \mathrm{kWh}_{\text {year }}{ }^{-1}$ for the lightweight thermal mass between 0.9 and $3.5 \mathrm{kWh}^{-1} \mathrm{year}^{-1}$ for the heavy thermal mass. It was, in general, very low compared to the energy needs, especially for the lightweight thermal mass.

One reason for the higher accuracy with the lightweight thermal mass could be the quasi-steady state calculation of the individual energy needs in Eqs. (2)-(4), which may be more accurate with a lightweight thermal mass.

An association was found between the RMSE and the outdoor air temperature following a power law. The corresponding coefficients of determination $R^{2}$ were 0.90718 and 0.76261 for the lightweight and heavy thermal mass, respectively, which indicates that almost
$91 \%$ and $76 \%$, respectively, of the variation in the $R M S E$ was associated with the outdoor air temperature.

The weaker association with the heavy thermal mass can be explained by the fact that with a heavy thermal mass, other climate factors have more influence on the nonlinearities, notably solar irradiation and the temperature difference between day and night.

The nonlinearity was also calculated for each climate. The ratio of the quadratic to the linear effects $Q L$ as function of the mean outdoor air temperature is illustrated in Fig. 4. Similarly, the ratio of the interaction to the linear effects $I L$ is illustrated in Fig. 5.

The results indicate that nonlinearities decreased, following a similar pattern, as a function of the outdoor air temperature.

In addition, for a given climate, when the ratio of the quadratic to the linear effects was above or below the fitted curve, the corresponding ratio of the interaction to the linear effects generally followed the same tendency.

It is interesting to observe that, for each climate, the ratio of the interaction to the linear effects was higher than the quadratic to the linear effects. Thus, despite the quadratic behavior, the interaction between the components had a greater effect on energy needs. In addition, little variation was observed in the ratios above $19{ }^{\circ} \mathrm{C}$, especially for the heavy thermal mass, indicating that the nonlinearities in thermal behavior become quasistable.

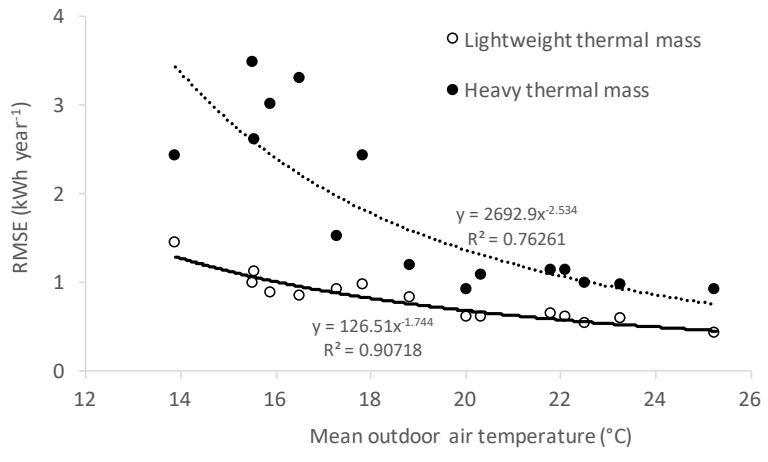

Fig. 3. RMSE of the metamodel for cooling energy needs versus the mean outdoor air temperature for the fifteen European climates. 


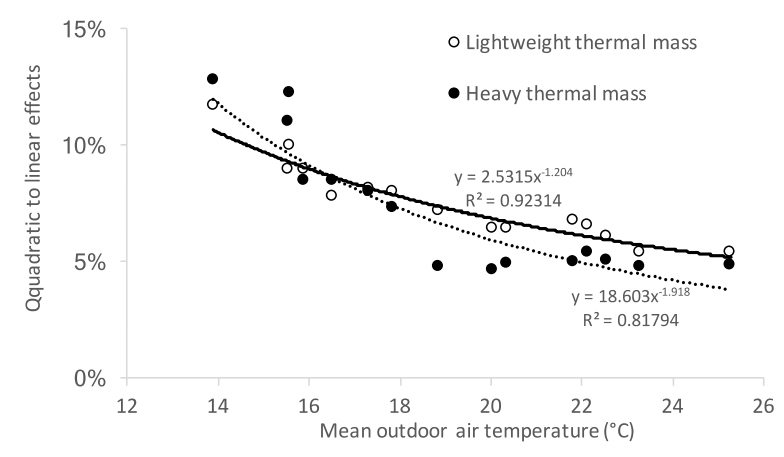

Fig. 4. Ratio of the quadratic to the linear effects versus mean outdoor air temperature for the fifteen European climates.

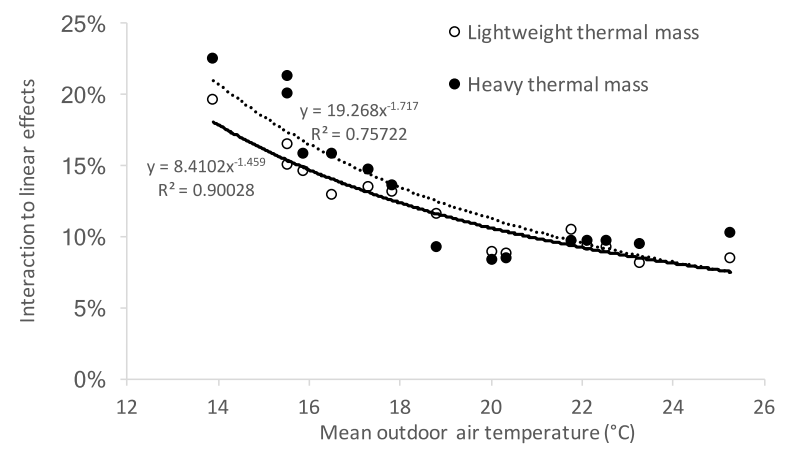

Fig. 5. Ratio of the interaction to the linear effects versus the mean outdoor air temperature for the fifteen European climates.

Furthermore, there was generally a strong association between the ratios and the outdoor air temperature, which varied between $76 \%$ and $96 \%$ depending on the measure. However, this temperature is not the only climate factor that impacted the nonlinearities in thermal behavior. The influence of other parameters such as the temperature difference between day and night, solar irradiation and wind speed could be significant. Further studies to investigate these effects would be interesting.

\subsection{Application to cold and hot climates}

\subsubsection{Cooling energy needs}

The metamodel was used to study the nonlinearities in thermal behavior in the cold climate of Helsinki and the hot climate of Athens with internal heat gains during occupation $p_{i g, o}$ varying from 5 to $40 \mathrm{~W} \mathrm{~m}^{-2}$.

The mean cooling energy needs as given by dynamic simulation versus internal heat gains are illustrated in Fig. 6 for both climates and for lightweight and heavy thermal masses. The variation of the energy needs as a function of $p_{i g, o}$ fitted almost perfectly with quadratic and linear polynomials for Helsinki and Athens, respectively.

It should be noticed that the heavy thermal mass reduced the cooling energy needs in Helsinki but increased them in Athens. The difference in energy needs was higher when the internal heat gains were intermediate in Helsinki and high in Athens.

The results also showed that, for Helsinki, when $p_{i g, o}$ are equal to $5 \mathrm{w} \mathrm{m}^{-2}$, the energy needs were close to zero for both thermal masses. Hence, in this case, the solar and internal heat gains were almost completely compensated by heat transfer by transmission and air change.

\subsubsection{Metamodel coefficients}

The metamodel coefficients were obtained by multiple regression analysis. A metamodel fit was achieved for each level of internal heat gains $p_{i g, o}$. However, the metamodel coefficients are presented only for the case where $p_{\text {ig,o }}$ were $20 \mathrm{~W} \mathrm{~m}^{-2}$. The coefficients for both climates and both thermal masses are shown in Table 3. These values are related to the metamodel with coded variables of the individual energy needs (varying from -1 to +1$)$.

The coefficient $a_{0}$ corresponds to the cooling energy needs when all the coded values of the individual energy needs are null, i.e., when they are equal to their mean level. Obviously, these energy needs were very high in the hot climate of Athens compared to the climate of Helsinki. In addition, at this level, the thermal mass reduced the energy needs in Helsinki and, conversely, increased them in Athens.

Furthermore, at this level, the thermal mass reduces the energy needs in Helsinki and conversely increases them in Athens. The coefficients $a_{1}, a_{2}$ and $a_{3}$, which correspond to the linear effects of the energy needs of heat transfer by transmission and air change $Q_{t r, o w}, Q_{t r, w}$ and $Q_{a c, i n f}$, were higher with a heavy thermal mass, in accordance with the fact that the reduction in cooling energy needs is more sensitive to heat loss with a heavy thermal mass.

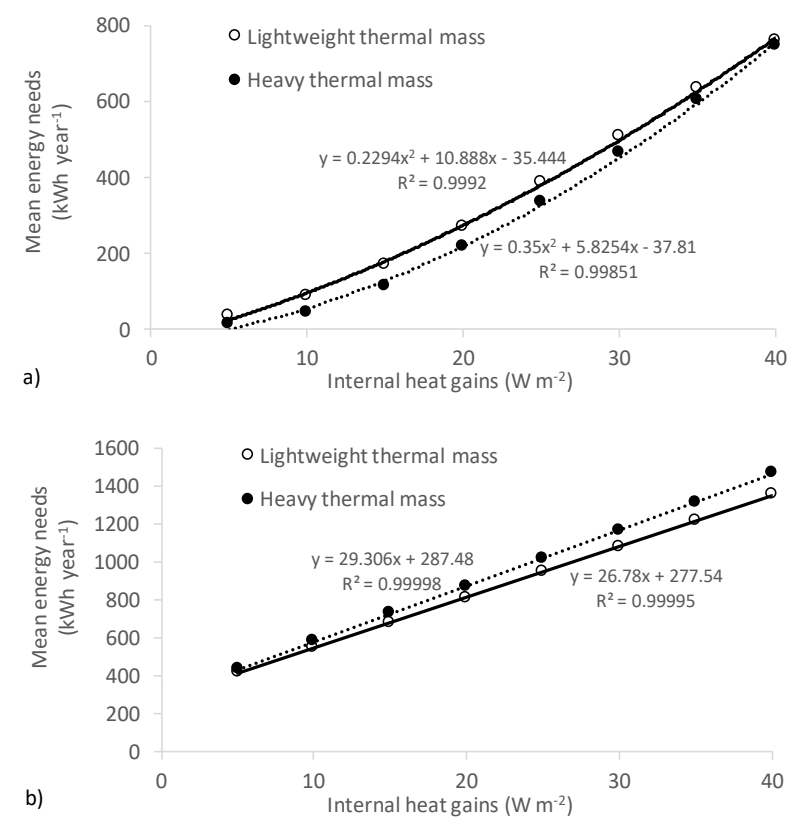

Fig. 6. Mean cooling energy needs as given by dynamic simulation versus internal heat gains: a) Helsinki and b) Athens.

Table 3. Coefficients of the metamodels using coded variables for Athens and Helsinki with internal heat gains of $20 \mathrm{~W} \mathrm{~m}^{-2}$. 


\begin{tabular}{|l|c|c|c|c|}
\hline Location & \multicolumn{2}{|c|}{ Helsinki } & \multicolumn{2}{c|}{ Athens } \\
\hline $\begin{array}{l}\text { Thermal } \\
\text { mass }\end{array}$ & Lightweight & Heavy & Lightweight & Heavy \\
\hline$a_{0}$ & 267.4 & 209.2 & 810.4 & 872.6 \\
\hline$a_{1}$ & -38.0 & -51.4 & -13.3 & -19.8 \\
\hline$a_{2}$ & -92.0 & -126.3 & -37.4 & -57.0 \\
\hline$a_{3}$ & -58.3 & -86.7 & -13.5 & -27.9 \\
\hline$a_{4}$ & 101.3 & 113.4 & 127.1 & 134.5 \\
\hline$a_{11}$ & 2.4 & 4.6 & 0.6 & 0.9 \\
\hline$a_{22}$ & 17.2 & 23.5 & 4.8 & 6.6 \\
\hline$a_{33}$ & 8.2 & 12.7 & 2.6 & 3.8 \\
\hline$a_{44}$ & 6.0 & 7.4 & 2.3 & 0.2 \\
\hline$a_{12}$ & 14.8 & 20.0 & 3.4 & 5.6 \\
\hline$a_{13}$ & 9.2 & 14.3 & 2.1 & 3.9 \\
\hline$a_{14}$ & -8.9 & -12.4 & -2.8 & -4.1 \\
\hline$a_{23}$ & 21.4 & 33.3 & 6.3 & 9.9 \\
\hline$a_{24}$ & -18.8 & -27.7 & -5.9 & -7.8 \\
\hline$a_{34}$ & -12.2 & -19.7 & -3.7 & -5.3 \\
\hline
\end{tabular}

In addition, all of each quadratic term had a positive effect $a_{i i}$, suggesting that the cooling energy needs varied as a convex function of the individual energy needs.

\subsubsection{Metamodel validation}

The metamodel fit was checked for each value of the internal heat gains $p_{i g, o}$ by comparing the results with those of TRNSYS software. The RMSE of the fits are shown in Fig. 7 for both climates and both thermal masses. As expected, the metamodels were more accurate for the hot climate of Athens than for the cold climate of Helsinki and for the lightweight thermal mass.

For Helsinki, the RMSE was relatively high when the internal heat gains were low, i.e. when the cooling energy needs were close to zero. However, when these gains were high, the RMSE was low, comparable to the RMSE obtained for Athens. In addition, the RMSE became stable, with approximately the same values for both thermal masses. Finally, the RMSE can be accurately expressed by a power regression, especially for the heavy thermal mass $\left(R^{2}=0.98092\right)$.

For Athens, a high level of agreement was observed between the results of the metamodel and the dynamic simulations, with errors below $0.5 \mathrm{kWh}$ year $^{-1}$ for the lightweight thermal mass and below $1.6 \mathrm{kWh}$ year $^{-1}$ for the heavy thermal mass. Furthermore, the RMSE for the lightweight thermal mass was almost constant. Although the RMSE increased linearly as a function of $p_{i g, o}$ for the heavy thermal mass $\left(\mathrm{R}^{2}=0.95338\right)$, the slop was 0.0255 $\mathrm{kWh}$ year ${ }^{-1} \mathrm{~W}^{-1} \mathrm{~m}^{2}$, which is less than $1 / 1000$ of that of the energy needs of $29.306 \mathrm{kWh}$ year ${ }^{-1} \mathrm{~W}^{-1} \mathrm{~m}^{2}$.

\subsubsection{Nonlinearity study}

The nonlinearity of thermal behavior was studied as a function of internal heat gains $p_{i g, o}$ using the measures of Eqs. (5-6).
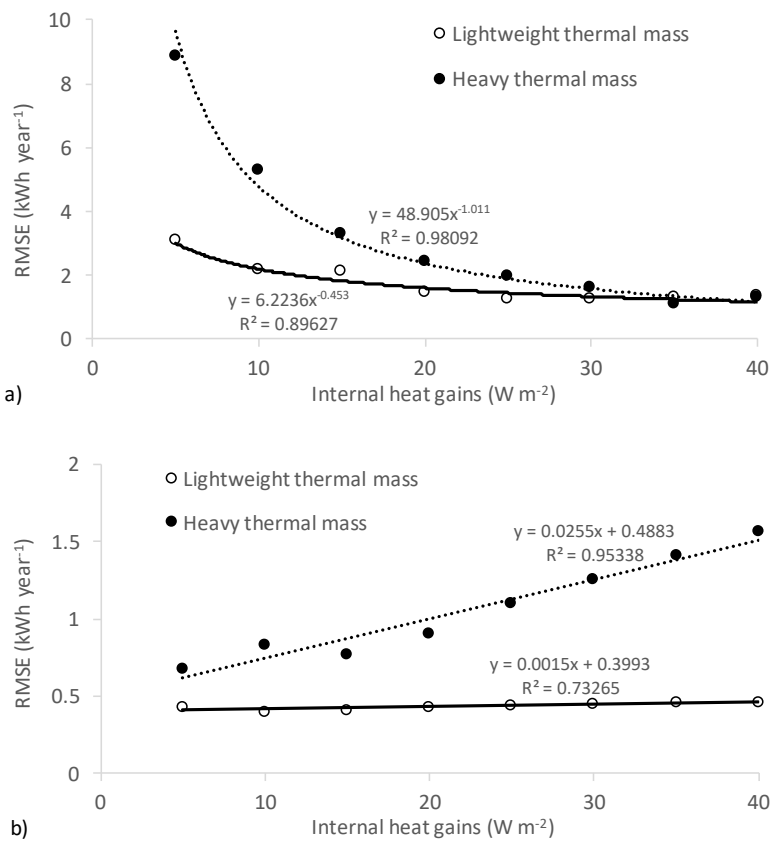

Fig. 7. RMSE of the metamodel for cooling energy needs versus internal heat gains: a) Helsinki and b) Athens.

The variations of the ratios of the quadratic to the linear effects $Q L$ (Eq. (5)) versus $p_{i g, o}$ are illustrated in Fig. 8 for both climates and both thermal masses.

For the cold climate of Helsinki, the results show that $Q L$ was high when $p_{i g, o}$ are equal to 5 and $10 \mathrm{~W} \mathrm{~m}^{-2}$, i.e. when the cooling energy needs were close to zero. In addition, they were strongly associated with $p_{i g, o}$ using power laws with coefficients of determination $R^{2}$ higher than 0.96 .

However, a better fit was again found for the lightweight thermal mass. Furthermore, when the internal gains were low, the quadratic behavior was more pronounced with a heavy thermal mass; above $20 \mathrm{~W} \mathrm{~m}^{-2}$ the difference was lower and even the behavior with a lightweight thermal mass was slightly more quadratic above $30 \mathrm{~W} \mathrm{~m}^{-2}$.

For the hot climate of Athens, there was a slight linear variation of $Q L$ varying from $4.8 \%$ to $6.6 \%$ for the lightweight thermal mass and from $4.0 \%$ to $6.2 \%$ for the heavy thermal. We deduced that the nonlinearities were low and almost stable in these conditions.

The ratios of the interaction to the linear effects $I L$ (Eq. (6)) versus $p_{i g, o}$ are illustrated in Figs. 9. For Helsinki, this ratio followed similar patterns to $Q L$. They were also strongly associated with $p_{i g, o}$ with coefficients of determination $R^{2}$ higher than 0.97 . For Athens, $I L$ were quasi-constant, with higher values for heavy thermal mass.

Finally, the ratio of the interaction to the linear effects $I L$ was higher than for quadratic to linear effects $Q L$ whatever the internal heat gains. This was also shown previously in relation to the mean outdoor air temperature of the climates. Thus, the interaction between the building components had a stronger effect than quadratic behavior. 

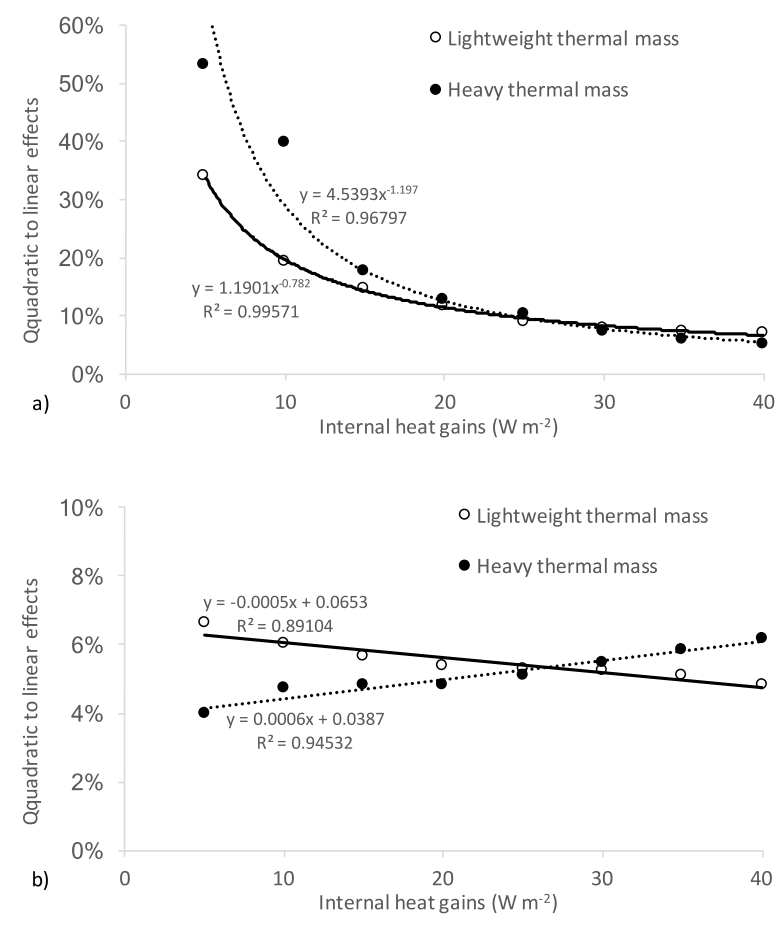

Fig. 8. Ratio of the quadratic to the linear effects versus internal heat gains: a) Helsinki and b) Athens.
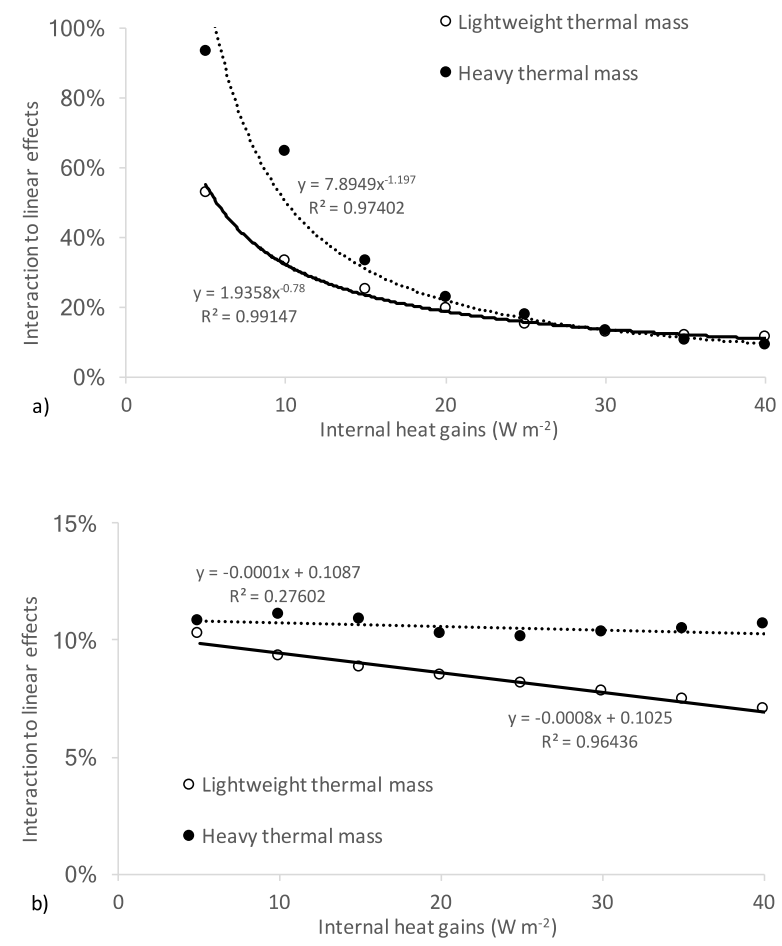

Fig. 9. Ratio of the interaction to the linear effects versus internal heat gains: a) Helsinki and b) Athens.

\section{Conclusion}

A metamodeling method to study nonlinearities in the thermal behavior of a building was presented. Two nonlinearity measures were introduced using the metamodel coefficients allowing to assess the relative importance of the quadratic and interaction effects in the metamodel.

The method was applied to the analysis of the nonlinearities in the thermal behavior of an office in relation to the mean outdoor air temperature of fifteen typical European climates and to different levels of internal heat gains for the coldest and hottest climates.

It was observed that metamodel errors generally decreased with mean outdoor air temperature and internal heat gains, i.e. when the cooling energy needs were high. When the climate was hot and internal heat gains were high, there was practically no difference between the results of the metamodel and dynamic simulation.

The nonlinearity of thermal behavior was accentuated when the climate was cold, with low internal heat gains, i.e. when energy needs were low and the metamodel errors high. The nonlinearity measures were accurately associated with the mean outdoor air temperature of the climates in accordance with decreasing power laws. Similar patterns were observed with internal heat gains for cold climates. However, for hot climates, the nonlinearities were quasi-stable, with a linear variation with respect to internal heat gains. It should be noticed that the interactions between the building components was found to be more influential on cooling energy needs than quadratic behavior.

Following the results of this study, we propose to classify building thermal behavior into three regimes: highly nonlinear close to zero energy needs; intermediate with decreasing nonlinearities that can be expressed by power functions; and, finally, a quasi-linear regime with almost-steady nonlinearities.

When the energy needs were close to zero, the thermal behavior was highly nonlinear with a relatively low metamodeling accuracy. The analysis of the nonlinearities in such conditions would require further investigation. For instance, alternative metamodels (e.g. artificial neural networks, radial basis functions, support vector machines, kriging) might produce more accurate approximations. However, some metamodels are computationally intensive and their coefficients would provide less insight than our metamodel that is based on a polynomial assumption.

Further metamodeling studies would be necessary to understand the nonlinearities in thermal behavior using metamodeling. In particular, the study of the influence of climate factors and energy systems is an interesting subject for research. In addition, it would be useful to identify the conditions in which nonlinear terms would be necessary to ensure the accuracy of simplified methods for use in future building standards.

\section{References}

1. J. P. C. Kleijnen, Statistical tools for simulation practitioners, Marcel Dekker, Inc., 1987.

2. T. W. Simpson, J. D. Poplinski, P. N. Koch and J. K. Allen, Metamodels for computer-based engineering design: Survey and recommendations, Engineering with computers 17 (2001) 2, 129-150. 
3. Y.-F. Li, S. H. Ng, M. Xie and T. N. Goh, A systematic comparison of metamodeling techniques for simulation optimization in decision support systems, Applied Soft Computing 10 (2010), 4, 1257-1273.

4. D. C. Montgomery, Design and analysis of experiments, Wiley, 2017.

5. E. Gratia and A. De Herde, A simple design tool for the thermal study of an office building, Energy and Buildings 34 (2002), 3, 279-289.

6. J. Xu, J.-H. Kim, H. Hong and J. Koo, A systematic approach for energy efficient building design factors optimization, Energy and Buildings 89 (2015), 8796.

7. R. Pino-Mejías, A. Pérez-Fargallo, C. Rubio-Bellido and J. Pulido-Arcas, Comparison of linear regression and artificial neural networks models to predict heating and cooling energy demand, energy consumption and co2 emissions, Energy 118 (2017), 24-36.

8. D. Shiming and J. Burnett, Energy use and management in hotels in hong kong, International Journal of Hospitality Management 21 (2002), 4, 371-380.

9. A. Aranda, G. Ferreira, M. D. Mainar-Toledo, S. Scarpellini and E. Llera Sastresa, Multiple regression models to predict the annual energy consumption in the spanish banking sector, Energy and Buildings 49 (2012), 380-387.

10. A. P. Melo, M. Fossati, R. S. Versage, M. J. Sorgato, V. A. Scalco and R. Lamberts, Development and analysis of a metamodel to represent the thermal behavior of naturally ventilated and artificially air-conditioned residential buildings, Energy and Buildings 112 (2016), 209221.

11. J. C. Lam, K. K. W. Wan, T. N. T. Lam and S. L. Wong, An analysis of future building energy use in subtropical hong kong, Energy 35 (2010), 3, 14821490.

12. A. Mastrucci, O. Baume, F. Stazi and U. Leopold, Estimating energy savings for the residential building stock of an entire city: A gis-based statistical downscaling approach applied to rotterdam, Energy and Buildings 75 (2014), 358367.

13. S. Karatasou, M. Santamouris and V. Geros, Modeling and predicting building's energy use with artificial neural networks: Methods and results, Energy and buildings 38 (2006), 8, 949-958.

14. M. Macas, F. Moretti, A. Fonti, A. Giantomassi, G. Comodi, M. Annunziato, S. Pizzuti and A. Capra, The role of data sample size and dimensionality in neural network based forecasting of building heating related variables, Energy and Buildings 111 (2016), 299-310.

15. F. Ascione, N. Bianco, C. De Stasio, G. M. Mauro and G. P. Vanoli, Artificial neural networks to predict energy performance and retrofit scenarios for any member of a building category: A novel approach, Energy 118 (2017), 999-1017.

16. B. Dong, C. Cao and S. E. Lee, Applying support vector machines to predict building energy consumption in tropical region, Energy and Buildings 37 (2005), 5, 545-553.

17. Q. Li, Q. Meng, J. Cai, H. Yoshino and A. Mochida, Predicting hourly cooling load in the building: A comparison of support vector machine and different artificial neural networks, Energy Conversion and Management 50 (2009), 1, 90-96.

18. Y. Heo and V. M. Zavala, Gaussian process modeling for measurement and verification of building energy savings, Energy and Buildings 53 (2012), 7-18.

19. X. Chen, H. Yang and K. Sun, Developing a metamodel for sensitivity analyses and prediction of building performance for passively designed highrise residential buildings, Applied energy 194 (2017), 422-439.

20. L. Van Gelder, H. Janssen and S. Roels, Metamodelling in robust low-energy dwelling design, 2nd Central European Symposium on Building Physics, Vienna University of Technology.

21. X. Gong, Y. Akashi and D. Sumiyoshi, Optimization of passive design measures for residential buildings in different chinese areas, Building and Environment 58 (2012), 46-57.

22. M. Rasouli, G. Ge, C. J. Simonson and R. W. Besant, Uncertainties in energy and economic performance of HVAC systems and energy recovery ventilators due to uncertainties in building and HVAC parameters, Applied Thermal Engineering 50 (2013), 1, 732-742.

23. J. Carlo and R. Lamberts, Development of envelope efficiency labels for commercial buildings: Effect of different variables on electricity consumption, Energy and Buildings 40 (2008), 11, 2002-2008.

24. D. G. Sanchez, B. Lacarrière, M. Musy and B. Bourges, Application of sensitivity analysis in building energy simulations: Combining first-and second-order elementary effects methods, Energy and Buildings 68 (2014), 741-750.

25. J. Maderspacher, P. Geyer, T. Auer and W. Lang, Comparison of different meta model approches with a detailed buiding model for long-term simulations, Conference Proceedings Building Simulation.

26. J. C. Lam, S. C. M. Hui and A. L. S. Chan, Regression analysis of high-rise fully airconditioned of fice buildings, Energy and Buildings 26 (1997), 2, 189-198.

27. I. Jaffal and C. Inard, A metamodel for building energy performance, Energy and Buildings 151 (2017), 501-510.

28. G. E. P. Box and D. W. Behnken, Some new three level designs for the study of quantitative variables, Technometrics 2 (1960), 4, 455-475. 